\title{
The effect of COVID-19 on emergency medical service call volumes and patient acuity: a cross-sectional study in Niagara, Ontario
}

\author{
Richard Ferron', Gina Agarwal ${ }^{2^{*}}$ (D), Rhiannon Cooper $^{2}$ (1) and Douglas Munkley ${ }^{1}$
}

\begin{abstract}
Background: The COVID-19 pandemic is a major public health problem. Subsequently, emergency medical services (EMS) have anecdotally experienced fluctuations in demand, with reports across Canada of both increased and decreased demand. Our primary objective was to assess the effect of the COVID-19 pandemic on call volumes for several determinants in Niagara Region EMS. Our secondary objective was to assess changes in paramedic-assigned patient acuity scores as determined using the Canadian Triage and Acuity Scale (CTAS).

Methods: We analyzed data from a regional EMS database related to call type, volume, and patient acuity for January to May 2016-2020. We used statistical methods to assess differences in EMS calls between 2016 and 2019 and 2020.

Results: A total of 114,507 EMS calls were made for the period of January 1 to May 26 between 2016 and 2020, inclusive. Overall, the incidence rate of EMS calls significantly decreased in 2020 compared to the total EMS calls in 2016-2019. Motor vehicle collisions decreased in 2020 relative to 2016-2019 (17\%), while overdoses relatively increased (70\%) in 2020 compared to 2016-2019. Calls for patients assigned a higher acuity score increased (CTAS 1) $(4.1 \%$ vs. $2.9 \%)$.

Conclusion: We confirmed that overall, EMS calls have decreased since the emergence of COVID-19. However, this effect on call volume was not consistent across all call determinants, as some call types rose while others decreased. These findings indicate that COVID-19 may have led to actual changes in emergency medical service demand and will be of interest to other services planning for future pandemics or further waves of COVID-19.
\end{abstract}

Keywords: Emergency medicine, Emergency medical services, COVID-19, Health services

\section{Background}

The global outbreak of severe acute respiratory syndrome coronavirus 2 (SARS-CoV-2), the virus that causes coronavirus disease 2019 (COVID-19), is a major public health problem. The first case of

\footnotetext{
* Correspondence: gina.agarwal@gmail.com

${ }^{2}$ Department of Family Medicine and Health Research Methods, Evidence and Impact, McMaster University, Hamilton, Canada

Full list of author information is available at the end of the article
}

COVID-19 in Canada was documented on January 25th, 2020 in Ontario [1]. Since then, Ontario has seen a large COVID-19 outbreak and at the time of writing is continuing to experience a rise in the number of positive cases [1]. As the impact of the COVID-19 pandemic on health care systems has become a concern in Ontario, paramedic services have begun to plan for anticipated impacts. Planning of this nature involves the ongoing monitoring of call

(c) The Author(s). 2021 Open Access This article is licensed under a Creative Commons Attribution 4.0 International License, which permits use, sharing, adaptation, distribution and reproduction in any medium or format, as long as you give appropriate credit to the original author(s) and the source, provide a link to the Creative Commons licence, and indicate if changes were made. The images or other third party material in this article are included in the article's Creative Commons licence, unless indicated otherwise in a credit line to the material. If material is not included in the article's Creative Commons licence and your intended use is not permitted by statutory regulation or exceeds the permitted use, you will need to obtain permission directly from the copyright holder. To view a copy of this licence, visit http://creativecommons.org/licenses/by/4.0/ The Creative Commons Public Domain Dedication waiver (http://creativecommons.org/publicdomain/zero/1.0/) applies to the data made available in this article, unless otherwise stated in a credit line to the data. 
volumes and types to anticipate future resource needs. There has been mixed information concerning the demand for emergency medical services (EMS). Perceptions abound that EMS will experience potential surges, during which containment is challenging yet crucial to the success of the health system response $[2,3]$. These are countered by anecdotes that EMS calls have decreased, as people are avoiding involvement with the health system for fear of infection [4].

Volumes of EMS calls are important to understand since they impact the response times, and are part of quality of EMS care [5]. Fluctuations in call volumes can result in more or less EMS resources available to attend to emergencies and could affect patient outcomes [6]. Therefore, in order to adequately plan and assist other EMS in Ontario and across Canada with similar planning, problems, and questions, it is important to understand the precise effect of the pandemic on EMS. Niagara EMS (NEMS) provides emergency medical services for an area in Southern Ontario with a population of about half a million people [7]. The average age of the population is 44 years old, with $64 \%$ of the population falling into the category of being 15 to 64 years of age; $48 \%$ are male; $89 \%$ are of European descent; $4 \%$ are Indigenous peoples; between 1 and $11 \%$ describe themselves as having non-European heritage (e.g. Asian); median post-tax family income is $\$ 72,105$ and single-parent family income is $\$ 46,684$ [7].

NEMS is a large service that, in June of 2016, had approximately $3.9 \%$ of Ontario's EMS call volumes [8]. Niagara region operates both an ambulance communications service with a dispatch centre, and a land ambulance service providing primary and advanced care paramedic services. There are approximately 343 full and part time paramedics in employment with the service, and 33 ambulances staffed at peak. These characteristics allow for reasonable comparisons to be made with other EMS service in Ontario. Though EMS in Ontario appears to have been affected by the COVID-19 pandemic, the specific impact of COVID19 on Ontario EMS has not yet been critically and robustly examined. The following is an assessment of the change in call types and acuity before and during the first wave of COVID-19.

\section{Methods}

\section{Study design}

A cross-sectional analysis of de-identified data obtained from the NEMS database (EDGE) was conducted. Electronic Patient Care Record (ePCR) data for each patient was extracted from the database. A time period of January 1 to May 26, 2020 only was chosen to reflect the onset and establishment phases of the pandemic. Further data from the same time period from 2016 to 2019 were extracted. These time periods were chosen to be comparable due to seasonal variation in EMS calls at the daily, monthly, and yearly level [9]. The aim of this study was to examine the impact of the ongoing COVID-19 pandemic on EMS call volumes, determinants (types of calls), and patient acuity at scene of arrival. Research ethics board approval was sought from the Hamilton Integrated Research Ethics Board, a partnership of McMaster University, St. Joseph's Healthcare Hamilton, and Hamilton Health Sciences; the project was deemed not to require ethics review because it was a quality improvement project using aggregate data, and an exemption was granted.

\section{Setting}

NEMS serves 12 municipalities and $1850 \mathrm{~km}^{2}$ that comprise the Niagara Region of Ontario [10]. As of the 2016 census, the population served by NEMS consisted of 447,888 people [7].

\section{Data collation}

The NEMS ambulance communications center utilizes the Medical Priority Dispatch System $\left(\mathrm{MPDS}^{\circ}\right)$ to categorize and triage calls coming into the center. Paramedic service data from both land ambulance and dispatch were accessed through a business intelligence solutions product called SAP Business Objects Edge Edition' EDGE [11]. Information regarding call type was extracted from the computer aided dispatch (CAD) software, in addition to the ePCR data pertaining to each patient.

\section{Measures}

EMS calls were reported as counts and were stratified by call determinant type (e.g. abdominal pain). We examined changes in patient acuity levels from January 1 2019 to May 26 2020. Patient acuity was measured through the Canadian Triage and Acuity Scale (CTAS), a validated 5-level triage score used by paramedics on scene arrival [12]. Level 1 indicates a highly urgent situation while level 5 indicates a non-urgent situation.

\section{Data analysis}

The total number of EMS calls were tabulated for each determinant and year, and then summed for the time period of 2016-2019. Chi-square tests of independence were conducted to compare the proportion of EMS calls in 2020 to EMS calls in 2016-2019 for each call determinant. Fisher's exact test was used for determinants with frequencies of 5 EMS calls or less. We also conducted a chi-square test of independence to compare the incidence rate ratios of EMS calls in 2020 to 2016-2019. Using population data for 2016, 2017, and 2019 in the Niagara region estimated by Statistics Canada [13], we 
made our own estimate of the population growth rate to calculate the projected population of Niagara in 2018 and 2020 using the following formulas:

$$
k=\frac{P_{t}-P_{t_{o}}}{P_{t_{o}} *\left(t-t_{o}\right)}
$$

where $\mathrm{k}$ is the population growth rate, $\mathrm{P}_{\mathrm{t}}$ is the population size in 2019, $P_{t_{o}}$ is the population size in 2017, and $\mathrm{t}$ and $\mathrm{t}_{\mathrm{o}}$ represent 2019 and 2017, respectively.

$$
P_{t}=P_{o} e^{k\left(t-t_{o}\right)}
$$

where $P_{t_{o}}$ is the population of Niagara in $2017, \mathrm{k}$ is the population growth rate, and $t$ and $t_{o}$ represent 2020 (and 2018) and 2017, respectively.

We used these projections to calculate the incidence rate ratios of EMS calls in 2016-2019 and in 2020, with person-years as the denominator and number of EMS calls as the numerator.

Changes in patient acuity were assessed for the timeframe of January $1^{\text {st }}, 2019$ to May $26^{\text {th }}, 2020$ using chisquare tests of independence to compare total calls in 2016-2019 to the total calls in 2020.

Data were analyzed in RStudio desktop version 1.2.50331 using $\mathrm{R}$ version 3.6.1 [14].

\section{Results}

A total of 114,507 EMS calls were made to NEMS for the period of January 1 to May 26 between 2016 and 2020, inclusive (Table 1). Of the total calls from 2016 to 2020, determinants representing a larger absolute proportion of all calls include sick person (16.8\%), falls (15.4\%), breathing problems (11.2\%), and chest pain (8.9\%). Overall, the incidence rate of EMS calls per person-year decreased in 2020 compared to 2016-2019.

\section{Call volumes and type}

Several types of EMS calls increased during JanuaryMay 2020 when compared to the same time frame in 2016-2019. Niagara had its first COVID-19 case on March $13^{\text {th }}, 2020$; therefore, EMS call volumes were most likely minimally affected in January and February. EMS calls significantly increased $(p<0.01)$ for cardiac arrest $(1.8 \%$ versus $1.4 \%)$, heart problems $(1.9 \%$ versus $1.7 \%)$, overdose/poisoning (4.4\% versus $2.6 \%$ ), pregnancy ( $0.5 \%$ versus $0.4 \%)$, stroke (3.3\% versus $2.9 \%)$, and unconscious (9.7\% versus $8.6 \%)$; see Table 1 .

However, compared to 2016-2019, EMS calls significantly decreased $(p<0.05)$ in 2020 for breathing problems (10.2\% versus $11.5 \%)$, carbon monoxide/ HAZMAT $(0.2 \%$ versus $0.3 \%)$, choking $(0.2 \%$ versus $0.3 \%)$, chest pain $(8.8 \%$ versus $9.0 \%)$, diabetic $(0.9 \%$ versus $1.2 \%$ ), sick person ( $11.3 \%$ versus $18.3 \%)$, motor vehicle collision (3.2\% versus 3.8\%), and unknown problem (4.1\% versus $5.1 \%)$.

The incidence rate of EMS calls in Niagara 2020 was significantly lower $(p<0.01)$ than EMS calls for the same time-period in 2016-2019 (0.046 versus 0.049 ); see Table 2.

\section{Patient acuity}

EMS calls significantly increased $(p<0.01)$ for calls assigned a CTAS level of $1(4.0 \%$ versus $2.9 \%)$ and for CTAS level 4 (14.3\% versus $12.3 \%)$; see Table 3. EMS calls significantly decreased $(p<0.01)$ for calls assigned a CTAS level of 2 (22.9\% versus $24.3 \%)$ and CTAS level 3 (78.4\% versus $94.1 \%)$.

\section{Discussion}

In our study we found that overall, the incidence rate of EMS calls from Jan-May 2020 significantly decreased compared to EMS calls made during the same timeframe in 2016-2019. This is in keeping with anecdotal reports in Canada describing a decrease in EMS calls beginning in April 2020 [4, 15]. In the U.S, a survey found that people were abstaining from calling 9-1-1, regardless of the severity of the health event, due to fear of COVID19 [16]. A serious consequence of this behaviour of avoiding the ED is that high acuity patients are waiting to seek necessary care until they are too unwell to be treated adequately [17].

We found that call volumes for several determinants increased, such as 9-1-1 calls for cardiac arrest, stroke, and heart attacks. Evidence exists in the literature to support the finding of increased cardiac arrests. Data from Northern Italy shows that during the pandemic, out-of-hospital cardiac arrests has increased by $58.0 \%$ [18]. A New York study has found that the incidence of out-of-hospital cardiac arrests needing resuscitation has tripled in 2020 compared to the same time frames in 2019 [19]. Other US data shows that 911 calls for cardiac arrest rose in March by $45.0 \%$, likely because people have been waiting too long before seeking care [17]. Indeed, fear of contracting COVID-19 may have contributed to EMS avoidance, even for patients experiencing life-threatening health events, resulting in the apparent increase in cardiac events and stroke. There is some evidence to suggest that our findings of increased cardiac arrests could also be related to SARS-CoV-2 itself. A systematic review found a correlation between COVID19 and cardiovascular complications, despite COVID-19 being a respiratory disease [20]. However, we were not able to examine if any of the patients presenting with cardiac arrest or heart attack were diagnosed with COVID-19.

In addition to negatively impacting physical health, the COVID-19 pandemic has also been a threat to mental 
Table 1 Five Year comparison of incidents and call types, January-May 2016-2020

\begin{tabular}{|c|c|c|c|c|c|c|c|c|c|}
\hline $\begin{array}{l}\text { EMS Call Type } \\
\text { and } \\
\text { Determinant } \\
\text { Number }\end{array}$ & $\begin{array}{l}\text { EMS } \\
\text { Calls } \\
2016 \mathrm{n} \\
(\%)\end{array}$ & $\begin{array}{l}\text { EMS } \\
\text { Calls } \\
2017 \mathrm{n} \\
(\%)\end{array}$ & $\begin{array}{l}\text { EMS } \\
\text { Calls } \\
2018 \mathrm{n} \\
(\%)\end{array}$ & $\begin{array}{l}\text { EMS } \\
\text { Calls } \\
2019 \mathrm{n} \\
(\%)\end{array}$ & $\begin{array}{l}\text { EMS } \\
\text { Calls } \\
2020 \mathrm{n} \\
(\%)\end{array}$ & $\begin{array}{l}\text { 4-Year } \\
\text { Average of } \\
\text { EMS Calls } \\
(2016-2019)\end{array}$ & $\begin{array}{l}\text { Proportion of } \\
\text { calls in } 2020 \text { vs. } \\
\text { calls } 2016-2019\end{array}$ & $\begin{array}{l}\text { Relative Change } \\
\text { in Calls (2020 vs. } \\
2016-2019)\end{array}$ & $\begin{array}{l}\text { Chi Square Test of } \\
\text { Independence } \\
\text { (Chi-score, } p \text {-value) }\end{array}$ \\
\hline Abdominal Pain & 784 (3.7) & $784(3.5)$ & 806 (3.3) & 885 (3.6) & 808 (3.6) & $814.75(3.5)$ & $3.8 \%$ vs. $3.7 \%$ & $3 \%$ & $\begin{array}{l}x^{2}(1)=0.59 \\
p=0.44\end{array}$ \\
\hline Allergies & $167(0.8)$ & $173(0.8)$ & $174(0.7)$ & $175(0.7)$ & $159(0.7)$ & $172.25(0.8)$ & $0.7 \%$ vs. $0.8 \%$ & $-4 \%$ & $\begin{array}{l}x^{2}(1)=0.23 \\
p=0.63\end{array}$ \\
\hline $\begin{array}{l}\text { Animal Bites/ } \\
\text { Attacks }\end{array}$ & $24(0.1)$ & $24(0.1)$ & $27(0.1)$ & $22(0.1)$ & $32(0.1)$ & $24.25(0.1)$ & $0.1 \%$ vs. $0.1 \%$ & $37 \%$ & $\begin{array}{l}x^{2}(1)=2.42 \\
p=0.12\end{array}$ \\
\hline $\begin{array}{l}\text { Assault/ } \\
\text { Sexual Assault }\end{array}$ & 695 (3.3) & $710(3.1)$ & $671(2.8)$ & $629(2.6)$ & $682(3.1)$ & $676.25(2.9)$ & $3.2 \%$ vs. $3.0 \%$ & $4 \%$ & $\begin{array}{l}x^{2}(1)=1.22 \\
p=0.27\end{array}$ \\
\hline Back Pain & $231(1.1)$ & $352(1.6)$ & $370(1.5)$ & 367 (1.5) & $304(1.4)$ & $330(1.4)$ & $1.4 \%$ vs. $1.5 \%$ & $-4 \%$ & $\begin{array}{l}x^{2}(1)=0.49 \\
p=0.47\end{array}$ \\
\hline $\begin{array}{l}\text { Breathing } \\
\text { Problems }\end{array}$ & $\begin{array}{l}2297 \\
(10.9)\end{array}$ & $\begin{array}{l}2385 \\
(10.6)\end{array}$ & $\begin{array}{l}2521 \\
(10.4)\end{array}$ & $\begin{array}{l}2310 \\
(9.5)\end{array}$ & $\begin{array}{l}2052 \\
(9.2)\end{array}$ & $\begin{array}{l}2378.25 \\
(10.3)\end{array}$ & $10.2 \%$ vs. $11.5 \%$ & $-12 \%$ & $\begin{array}{l}x^{2}(1)=22.53 \\
p<0.01^{*}\end{array}$ \\
\hline $\begin{array}{l}\text { Burns/ } \\
\text { Explosions }\end{array}$ & $37(0.2)$ & $35(0.2)$ & $62(0.3)$ & $72(0.3)$ & $51(0.2)$ & $51.5(0.2)$ & $0.2 \%$ vs. $0.2 \%$ & $4 \%$ & $\begin{array}{l}x^{2}(1)=0.033 \\
p=0.86\end{array}$ \\
\hline $\begin{array}{l}\text { Carbon } \\
\text { Monoxide/ } \\
\text { HAZMAT }\end{array}$ & $65(0.3)$ & $67(0.3)$ & $78(0.3)$ & $75(0.3)$ & $47(0.2)$ & $71.25(0.3)$ & $0.2 \%$ vs. $0.3 \%$ & $-31 \%$ & $\begin{array}{l}x^{2}(1)=5.85 \\
\boldsymbol{p}=\mathbf{0 . 0 1 6 ^ { * }}\end{array}$ \\
\hline Cardiac Arrest & $255(1.2)$ & $321(1.4)$ & $369(1.5)$ & $336(1.4)$ & $385(1.7)$ & $320.25(1.4)$ & $1.8 \%$ vs. $1.4 \%$ & $26 \%$ & $\begin{array}{l}x^{2}(1)=14.89 \\
\boldsymbol{p}<0.01^{*}\end{array}$ \\
\hline Chest Pain & $\begin{array}{l}1798 \\
(8.5)\end{array}$ & $\begin{array}{l}1765 \\
(7.8)\end{array}$ & $\begin{array}{l}2076 \\
(8.6)\end{array}$ & $\begin{array}{l}2007 \\
(8.2)\end{array}$ & $\begin{array}{l}1790 \\
(8.1)\end{array}$ & $1911.5(8.3)$ & $8.8 \%$ vs. $9.0 \%$ & $-3 \%$ & $\begin{array}{l}x^{2}(1)=1.21 \\
p=0.27\end{array}$ \\
\hline Choking & $58(0.3)$ & $76(0.3)$ & $78(0.3)$ & $104(0.4)$ & $54(0.2)$ & $79(0.3)$ & $0.2 \%$ vs. $0.3 \%$ & $-29 \%$ & $\begin{array}{l}x^{2}(1)=5.48 \\
p=0.019^{*}\end{array}$ \\
\hline Convulsions & 731 (3.5) & $784(3.5)$ & 778 (3.2) & $814(3.3)$ & 730 (3.3) & 776.75 (3.4) & $3.4 \%$ vs. $3.5 \%$ & $-3 \%$ & $\begin{array}{l}x^{2}(1)=0.35 \\
p=0.55\end{array}$ \\
\hline Diabetic & 278 (1.3) & $268(1.2)$ & $268(1.1)$ & $249(1.0)$ & $203(0.9)$ & $265.75(1.2)$ & $0.9 \%$ vs. $1.2 \%$ & $-21 \%$ & $\begin{array}{l}x^{2}(1)=9.26 \\
\boldsymbol{p}<0.01^{*}\end{array}$ \\
\hline $\begin{array}{l}\text { Drowning/ } \\
\text { Diving Accident }\end{array}$ & $11(0.1)$ & $<5$ & $8(0.0)$ & $9(0.0)$ & $6(0.0)$ & $8(0.0)$ & $0.0 \%$ vs.0 $.0 \%$ & $-23 \%$ & $p=0.69$ \\
\hline $\begin{array}{l}\text { Electrocution/ } \\
\text { Lightning }\end{array}$ & $5(0.0)$ & $<5$ & $<5$ & $<5$ & $<5$ & $<5$ & $0.0 \%$ vs. $0.0 \%$ & $-74 \%$ & $p=0.22$ \\
\hline $\begin{array}{l}\text { Eye Problems/ } \\
\text { Injuries }\end{array}$ & $27(0.1)$ & $24(0.1)$ & $21(0.1)$ & $20(0.1)$ & $23(0.1)$ & $23(0.1)$ & $0.1 \%$ vs. $0.1 \%$ & $3 \%$ & $\begin{array}{l}x^{2}(1)=0.027 \\
p=0.87\end{array}$ \\
\hline Falls & $\begin{array}{l}2715 \\
(12.9)\end{array}$ & $\begin{array}{l}3050 \\
(13.5)\end{array}$ & $\begin{array}{l}3277 \\
(13.5)\end{array}$ & $\begin{array}{l}3248 \\
(13.3)\end{array}$ & $\begin{array}{l}3001 \\
(13.5)\end{array}$ & 3072.5 (13.3) & $15.6 \%$ vs. $15.4 \%$ & $2 \%$ & $\begin{array}{l}x^{2}(1)=0.59 \\
p=0.44\end{array}$ \\
\hline Headache & $145(0.7)$ & $127(0.6)$ & $115(0.5)$ & $155(0.6)$ & $146(0.7)$ & $135.5(0.6)$ & $0.7 \%$ vs. $0.6 \%$ & $11 \%$ & $\begin{array}{l}x^{2}(1)=1.47 \\
p=0.23\end{array}$ \\
\hline Heart Problems & 395 (1.9) & $314(1.4)$ & $370(1.5)$ & $424(1.7)$ & $423(1.9)$ & $375.75(1.6)$ & $1.9 \%$ vs. $1.7 \%$ & $17 \%$ & $\begin{array}{l}x^{2}(1)=8.24 \\
\boldsymbol{p}=0.0041^{*}\end{array}$ \\
\hline $\begin{array}{l}\text { Heat/Cold } \\
\text { Exposure }\end{array}$ & $19(0.1)$ & $19(0.1)$ & $30(0.1)$ & $40(0.2)$ & $34(0.2)$ & $27(0.1)$ & $0.2 \%$ vs. $0.1 \%$ & $33 \%$ & $\begin{array}{l}x^{2}(1)=1.88 \\
p=0.17\end{array}$ \\
\hline $\begin{array}{l}\text { Hemorrhage/ } \\
\text { Laceration }\end{array}$ & 790 (3.8) & 859 (3.8) & 946 (3.9) & 898 (3.7) & $826(3.7)$ & 873.25 (3.8) & $3.9 \%$ vs. $3.9 \%$ & $-2 \%$ & $\begin{array}{l}x^{2}(1)=0.21 \\
p=0.64\end{array}$ \\
\hline $\begin{array}{l}\text { Inaccessible } \\
\text { Incident }\end{array}$ & $<5$ & $<5$ & $<5$ & $<5$ & $5(0.0)$ & $2.75(0.0)$ & $0.0 \%$ vs. $0.0 \%$ & $90 \%$ & $p=0.22$ \\
\hline $\begin{array}{l}\text { Overdose/ } \\
\text { Poisoning }\end{array}$ & $416(1.9)$ & $503(2.2)$ & $548(2.3)$ & $865(3.5)$ & $928(4.2)$ & $583(2.3)$ & $4.4 \%$ vs. $2.6 \%$ & $70 \%$ & $\begin{array}{l}x^{2}(1)=176.49 \\
\boldsymbol{p}<0.01^{*}\end{array}$ \\
\hline Pregnancy & $83(0.4)$ & $90(0.4)$ & $90(0.4)$ & $108(0.4)$ & $105(0.5)$ & $92.75(0.4)$ & $0.5 \%$ vs. $0.4 \%$ & $18 \%$ & $\begin{array}{l}x^{2}(1)=11.80 \\
\boldsymbol{p}<\mathbf{0 . 0 1 *}\end{array}$ \\
\hline $\begin{array}{l}\text { Psychiatric/ } \\
\text { Abnormal }\end{array}$ & $\begin{array}{l}1284 \\
(6.1)\end{array}$ & $\begin{array}{l}1467 \\
(6.5)\end{array}$ & $\begin{array}{l}1529 \\
(6.3)\end{array}$ & $\begin{array}{l}1588 \\
(6.5)\end{array}$ & $\begin{array}{l}1426 \\
(6.4)\end{array}$ & $1467(6.4)$ & $6.9 \%$ vs. $6.8 \%$ & $1 \%$ & $\begin{array}{l}x^{2}(1)=0.12 \\
p=0.73\end{array}$ \\
\hline
\end{tabular}


Table 1 Five Year comparison of incidents and call types, January-May 2016-2020 (Continued)

\begin{tabular}{|c|c|c|c|c|c|c|c|c|c|}
\hline $\begin{array}{l}\text { EMS Call Type } \\
\text { and } \\
\text { Determinant } \\
\text { Number }\end{array}$ & $\begin{array}{l}\text { EMS } \\
\text { Calls } \\
2016 \mathrm{n} \\
(\%)\end{array}$ & $\begin{array}{l}\text { EMS } \\
\text { Calls } \\
2017 \mathrm{n} \\
(\%)\end{array}$ & $\begin{array}{l}\text { EMS } \\
\text { Calls } \\
2018 \mathrm{n} \\
(\%)\end{array}$ & $\begin{array}{l}\text { EMS } \\
\text { Calls } \\
2019 \mathrm{n} \\
(\%)\end{array}$ & $\begin{array}{l}\text { EMS } \\
\text { Calls } \\
2020 \mathrm{n} \\
(\%)\end{array}$ & $\begin{array}{l}\text { 4-Year } \\
\text { Average of } \\
\text { EMS Calls } \\
(2016-2019)\end{array}$ & $\begin{array}{l}\text { Proportion of } \\
\text { calls in } 2020 \text { vs. } \\
\text { calls } 2016-2019\end{array}$ & $\begin{array}{l}\text { Relative Change } \\
\text { in Calls (2020 vs. } \\
2016-2019)\end{array}$ & $\begin{array}{l}\text { Chi Square Test of } \\
\text { Independence } \\
\text { (Chi-score, } p \text {-value) }\end{array}$ \\
\hline Sick Person & $\begin{array}{l}3362 \\
(15.9)\end{array}$ & $\begin{array}{l}3589 \\
(15.9)\end{array}$ & $\begin{array}{l}3797 \\
(15.7)\end{array}$ & $\begin{array}{l}3495 \\
(14.3)\end{array}$ & $\begin{array}{l}2255 \\
(10.2)\end{array}$ & $\begin{array}{l}3560.75 \\
(15.4)\end{array}$ & $11.3 \%$ vs. $18.2 \%$ & $-38 \%$ & $\begin{array}{l}X^{2}(1)=404.71 \\
p<0.01^{*}\end{array}$ \\
\hline Stab/Gunshot & $34(0.2)$ & $27(0.1)$ & $27(0.1)$ & $36(0.2)$ & $42(0.2)$ & $31(0.1)$ & $0.2 \%$ vs. $0.1 \%$ & $40 \%$ & $\begin{array}{l}x^{2}(1)=3.71 \\
p=0.054\end{array}$ \\
\hline Stroke & $619(2.9)$ & $635(2.8)$ & 707 (2.9) & $690(2.8)$ & 717 (3.2) & $662.75(2.9)$ & $3.3 \%$ vs. $3 . \%$ & $13 \%$ & $\begin{array}{l}x^{2}(1)=7.93 \\
\boldsymbol{p}<0.01^{*}\end{array}$ \\
\hline $\begin{array}{l}\text { Motor Vehicle } \\
\text { Collision }\end{array}$ & $788(3.7)$ & $837(3.7)$ & 881 (3.6) & $903(3.7)$ & $682(3.1)$ & $852.25(3.7)$ & $3.2 \%$ vs. $3.8 \%$ & $-17 \%$ & $\begin{array}{l}x^{2}(1)=20.18 \\
\boldsymbol{p}<0.01^{*}\end{array}$ \\
\hline Traumatic Injuries & $500(2.4)$ & $484(2.1)$ & $484(2.0)$ & 487 (1.9) & $403(1.8)$ & $488.75(2.1)$ & $1.8 \%$ vs. $2.2 \%$ & $-15 \%$ & $\begin{array}{l}x^{2}(1)=8.20 \\
\boldsymbol{p}=0.0042^{*}\end{array}$ \\
\hline Unconscious & $\begin{array}{l}1541 \\
(7.3)\end{array}$ & $\begin{array}{l}1689 \\
(7.5)\end{array}$ & $\begin{array}{l}2018 \\
(8.3)\end{array}$ & $\begin{array}{l}2033 \\
(8.3)\end{array}$ & $\begin{array}{l}1960 \\
(8.8)\end{array}$ & $1820.25(7.9)$ & $9.7 \%$ vs. $8.6 \%$ & $13 \%$ & $\begin{array}{l}x^{2}(1)=21.11 \\
\boldsymbol{p}<0.01^{*}\end{array}$ \\
\hline Unknown Problem & $902(4.3)$ & $\begin{array}{l}1111 \\
(4.9)\end{array}$ & $\begin{array}{l}1105 \\
(4.6)\end{array}$ & $\begin{array}{l}1369 \\
(5.6)\end{array}$ & $872(3.9)$ & $1121.75(4.9)$ & $4.1 \%$ vs. $5.1 \%$ & $-20 \%$ & $\begin{array}{l}x^{2}(1)=35.14 \\
p<0.01^{*}\end{array}$ \\
\hline Pandemic Protocol & 0 & 0 & 0 & 0 & $\begin{array}{l}1060 \\
(4.8)\end{array}$ & 0 & $5.0 \%$ vs. $0.0 \%$ & $3 \%$ & $p<0.01^{*}$ \\
\hline
\end{tabular}

*Indicates significance

health [21, 22]. Overdose/poisoning demonstrated the largest increase in call volumes than any other call determinant. One group at an elevated risk for experiencing the negative mental health impacts of the COVID-19 pandemic are those who used substances or are in recovery [23]. In order to control the spread of COVID19, multiple safe injection sites have remained closed during the pandemic. It is possible that these closures could have contributed to the alarming increase in overdoses/poisonings seen in the current study. Some safe injection sites in Ontario have reopened after noticing an increase in overdoses and noting the role that these sites play in harm reduction [24]. The interruption of regular primary health care and the switch to use of virtual care versus in-person care may also have impacted overdose numbers. Patients using prescription opioids may be monitored by urine testing less frequently and primary care may have been forced to be less vigilant while prescribing narcotics in these altered circumstances. Additionally, physical distancing measures may have led to an increase in social isolation and stress, increasing the risk of overdose for those who use substances due to lack of support and emotional strain. In support of this theory, lack of social support has been shown to predict non-fatal drug overdose in females, and mental health problems, such as depression, have been shown to predict overdose in both males and females [25]. It is worth noting that 9-1-1 calls for mental health appear to have decreased during the pandemic, although not significantly. Previously, 9-1-1 may have been called prior to an overdose, and those cases would have been identified as mental health issues. However, if patients are avoiding calling 9-1-1, there may be a subsequent increase in incidences of overdose.

Several call determinants decreased from January 2020 to May 2020. Unexpectedly, EMS calls regarding breathing problems and sick people decreased. An explanation for this could be the introduction of a new MPDS card on April 30, 2020; the 'pandemic protocol card'. Calls that would have previously been categorized as "sick person" or "breathing problems", the determinants associated with COVID-19, became categorized as 'pandemic protocol'. The pandemic protocol card was meant to provide an opportunity for an altered response to call

Table 2 Comparison of total calls per person-year in 2016-2019 to 2020

\begin{tabular}{|c|c|c|c|c|c|c|c|}
\hline & 2016 & 2017 & 2018 & 2019 & 2020 & $\begin{array}{l}\text { Average EMS calls per } \\
\text { person-year 2016-2019 }\end{array}$ & $\begin{array}{c}\text { Chi Square Test } \\
\text { of Independence } \\
\text { (Chi-score, } p \text {-value) }\end{array}$ \\
\hline \multirow[t]{2}{*}{$\begin{array}{l}\text { EMS calls per } \\
\text { person-year }\end{array}$} & 0.047 & 0.048 & 0.051 & 0.051 & 0.046 & 0.049 & $\begin{array}{c}x^{2}(1)=75.66 \\
p<0.01^{*}\end{array}$ \\
\hline & 2016 & 2017 & 2018 & 2019 & 2020 & & \\
\hline Population & 447,888 & 465,569 & 472,416 & 479,183 & 486,414 & & \\
\hline
\end{tabular}

*Indicates significance Population values for 2018 and 2020 were projected using the formula written in the methods section 
Table 3 Five Year comparison of CTAS Scores, January-May 2016-2020

\begin{tabular}{|c|c|c|c|c|c|c|c|c|c|}
\hline CTAS Level & $\begin{array}{l}\text { Total EMS } \\
\text { Calls in } \\
2016 \mathrm{n}(\%)\end{array}$ & $\begin{array}{l}\text { Total EMS } \\
\text { Calls in } \\
2017 \text { n (\%) }\end{array}$ & $\begin{array}{l}\text { Total EMS } \\
\text { Calls in } \\
2018 \mathrm{n}(\%)\end{array}$ & $\begin{array}{l}\text { Total EMS } \\
\text { Calls in } \\
2019 \text { n (\%) }\end{array}$ & $\begin{array}{l}\text { Total EMS } \\
\text { Calls in } \\
2020 \mathrm{n}(\%)\end{array}$ & $\begin{array}{l}\text { Total } \\
\text { Calls for } \\
\text { Each } \\
\text { Level }\end{array}$ & $\begin{array}{l}\text { Proportion of } \\
\text { calls in } 2020 \text { vs. } \\
\text { calls } 2016-2019\end{array}$ & $\begin{array}{l}\text { Relative } \\
\text { Change } \\
\text { in Calls }\end{array}$ & $\begin{array}{l}\text { Chi Square Test of } \\
\text { Independence } \\
\text { (Chi-score, } p \text {-value) }\end{array}$ \\
\hline 1 (Resuscitation) & $515(2.4)$ & $605(2.7)$ & 749 (3.0) & $773(3.1)$ & 894 (3.9) & 3536 (3.1) & $4.1 \%$ vs. $2.9 \%$ & $-41 \%$ & $\begin{array}{l}x^{2}(1)=70.36 \\
\boldsymbol{p}<0.01 *\end{array}$ \\
\hline 2 (Emergent) & 4509 (21.4) & 4491 (20.0) & 4656 (18.8) & 4550 (18.4) & 4261 (18.6) & $\begin{array}{l}22,467 \\
(19.4)\end{array}$ & $22.9 \%$ vs. $24.3 \%$ & $6 \%$ & $\begin{array}{l}x^{2}(1)=10.90 \\
\boldsymbol{p}=\mathbf{0 . 0 1 *}\end{array}$ \\
\hline 3 (Urgent) & $\begin{array}{l}10,996 \\
(52.1)\end{array}$ & $\begin{array}{l}11,137 \\
(49.7)\end{array}$ & $\begin{array}{l}11,513 \\
(46.5)\end{array}$ & $\begin{array}{l}11,457 \\
(46.3)\end{array}$ & $\begin{array}{l}10,060 \\
(43.9)\end{array}$ & $\begin{array}{l}55,163 \\
(47.6)\end{array}$ & $78.4 \%$ vs. $94.1 \%$ & $10 \%$ & $\begin{array}{l}X^{2}(1)=152.61 \\
\boldsymbol{p}<0.01^{*}\end{array}$ \\
\hline $\begin{array}{l}4 \text { (Less } \\
\text { Urgent) }\end{array}$ & $1935(9.2)$ & 2325 (10.4) & 2953 (11.9) & 2954 (11.9) & 2856 (12.5) & $\begin{array}{l}13,023 \\
(11.2)\end{array}$ & $14.3 \%$ vs. $12.3 \%$ & $-17 \%$ & $\begin{array}{l}x^{2}(1)=43.89 \\
\boldsymbol{p}<0.01^{*}\end{array}$ \\
\hline $\begin{array}{l}5 \text { (Non- } \\
\text { Urgent) }\end{array}$ & 2091 (9.9) & 2453 (10.9) & 3185 (12.9) & 3181 (12.8) & 2650(11.6) & $\begin{array}{l}13,560 \\
(11.7)\end{array}$ & $13.1 \%$ vs. $13.3 \%$ & $-1 \%$ & $\begin{array}{l}x^{2}(1)=0.069 \\
p=0.79\end{array}$ \\
\hline
\end{tabular}

*Indicates significance. Proportions were calculated using total calls and calls not assigned a CTAS number were not included in the table

types identified as potential COVID-19 cases during periods that threaten to overwhelm EMS resources. The protocol encompasses a variety of presentations, including shortness of breath and chest pain. For this reason, categorizing a patient in only one protocol would result in misclassification. Therefore, call volumes for breathing problems and sick person may not have decreased as much as we reported.

Of all determinants that experienced a decrease in call volumes, motor vehicle collisions (MVC) decreased the most. People may have been commuting and travelling less frequently and had been encouraged to stay at home, therefore were less likely to become involved in car accidents. A study in Michigan, USA, examining the effect of shelter-in-place orders on orthopaedic trauma, also found that motor vehicle collisions decreased by 17\% in March 2020 relative to March 2019 [26]. Although Ontario did not issue a shelter-in-place order, citizens were instructed to stay-at-home which may offer an explanation for the decrease in the number of MVC.

The number of patients presenting as high acuity (CTAS Level 1) and lower acuity (CTAS level 4) increased during the pandemic. A possible explanation for this is that lower acuity patients are not seeking care in time, so that when EMS is called they present as high acuity [16]. However, the increase in overdoses could also be contributing to higher patient acuity upon scene arrival. EMS calls for emergent (CTAS Level 2) and urgent (CTAS Level 3) situations have decreased from January 2019 to May 2020. This finding may also be explained by a fear of contracting COVID-19 in emergency rooms, and therefore avoiding EMS.

\section{Limitations}

We cannot solely attribute the decrease in EMS calls to the COVID-19 pandemic. EMS calls could also have decreased due to changes at dispatch because of an MPDS system upgrade that was implemented in 2019.
Additionally, in the same year the triage process was changed for certain conditions, a Mobile Integrated Health Team, with additional mental health training, may have been dispatched instead of a regular ambulance. The decrease in EMS calls may also be attributed to the lack of tourists in attendance in the region, which typically accounts for a portion of the Niagara EMS calls each year. The population of Niagara has 13 million visitors each year, and a decrease in visitors may lead to a decrease in 9-1-1 calls [27]. Though the current data were not collated on an urban vs. rural basis, it is likely that the drop in EMS calls is driven by urban regions, where tourism is high in the spring and summer. In addition, the rules for the classification of call determinants change every 1-2 years. However, we have confirmed that call determinants have mostly stayed the same, and any changes that were made would have not affected the selection of determinants chosen at the time of dispatch. There may also be variation between the call determinant assigned at the time of triage, based on the assessment of the paramedics. With regards to patient acuity, we cannot rule out the possibility that paramedic documented CTAS may not be completely accurate as it is a subjective measure.

\section{Conclusion}

We have confirmed that overall, EMS calls in the Niagara region have significantly decreased during the first 5 months of the COVID-19 pandemic, whilst some types increased. Specifically, EMS calls for overdose increased the most out of any other call determinant, while calls for motor vehicle collisions decreased more than any other determinant. Calls related to high patient acuity levels significantly increased, while EMS calls for urgent and emergent patients significantly decreased. Our findings suggest that the COVID-19 pandemic could have contributed to a decrease in EMS calls overall, and increased certain types of calls, including an 
increase in the number of high acuity patients. The results of this study may be generalizable to other geographical areas with similar population sizes and characteristics but may not be generalizable to areas that don't provide universal healthcare. These findings may be of interest to other EMS as they plan for future pandemics or second waves of COVID-19, including the ability to predict call type in an effort to adjust a targeted response. This information can also provide an opportunity for dialogue between EMS agencies and health care partners on the consequences of delays in seeking treatment.

\section{Abbreviations}

SARS-CoV-2: severe acute respiratory syndrome coronavirus 2; COVID19: coronavirus disease 2019; EMS: Emergency medical services; NEMS: Niagara emergency medical service; ePCR: electronic Patient Care Record; MPDS ${ }^{\oplus}$ : Medical priority dispatch system; CAD: Computer aided dispatch; CTAS: Canadian triage and acuity scale; MVC: Motor vehicle collisions

\section{Acknowledgements}

We would like to acknowledge Niagara Emergency Medical Services for providing data for this study.

\section{Authors' contributions}

RF provided data, imparted expertise in the field of emergency medicine, and developed the research question. GA provided methodological and analytical expertise and wrote a first draft of the manuscript. RC supported in the writing and data analysis process. DM provided field expertise. All authors read and approved of the final manuscript.

\section{Funding}

This research was not funded.

\section{Availability of data and materials}

The data that support the findings of this study are available from Niagara Emergency Medical Services, but restrictions apply to the availability of these data, which were used under license for the current study, and so are not publicly available. Data are however available from the authors upon reasonable request and with permission of Rick Ferron.

\section{Declarations}

\section{Ethics approval and consent to participate}

Hamilton Integrated Research ethics board [a partnership of McMaster University, St. Joseph's Healthcare Hamilton and Hamilton Health Sciences] approval was sought, the project was deemed not to require ethics review because it was a quality improvement project using aggregate data, and an exemption was granted.

\section{Consent for publication}

Not applicable.

\section{Competing interests}

The authors declare they have no competing interests.

\section{Author details}

${ }^{1}$ Niagara Emergency Medical Service, Niagara, Canada. ${ }^{2}$ Department of Family Medicine and Health Research Methods, Evidence and Impact, McMaster University, Hamilton, Canada.
Received: 23 September 2020 Accepted: 17 March 2021

Published online: 29 March 2021

\section{References}

1. Berry I, Soucy J-PR, Tuite A, Fisman D. Open access epidemiologic data and an interactive dashboard to monitor the COVID-19 outbreak in Canada. CMAJ. 2020;192(15):E420. https://doi.org/10.1503/cmaj.75262.

2. Wilder-Smith A, Freedman DO. Isolation, quarantine, social distancing and community containment: pivotal role for old-style public health measures in the novel coronavirus (2019-nCoV) outbreak. J Travel Med 2020;27(2):taaa020

3. Bedford J, Enria D, Giesecke J, Heymann DL, Ihekweazu C, Kobinger G, et al. COVID-19: towards controlling of a pandemic. Lancet. 2020;395(10229): 1015-8. https://doi.org/10.1016/S0140-6736(20)30673-5.

4. Calls for Hamilton paramedics down during COVID-19 pandemic, says chief. Global News. Available from: https://globalnews.ca/news/6887128/calls-ha milton-paramedics-down-coronavirus/. [cited 2020 Jun 9]

5. Ahn KO, Shin SD, Cha WC, Jun C, Lee TS, Pirrallo RG. A model for the association of the call volume and the unavailable-for-response interval on the delayed ambulance response for out-of-hospital cardiac arrest using a geographic information system. Prehosp Emerg Care. 2010;14(4):469-76. https://doi.org/10.3109/10903127.2010.497895.

6. Wilde ET. Do emergency medical system response times matter for health outcomes? Health Econ. 2013;22(7):790-806. https://doi.org/10.1002/hec.2 851.

7. Government of Canada SC. Census Profile, 2016 Census - Niagara, Regional municipality [Census division], Ontario and Ontario [Province]. 2017. Available from: https://www12.statcan.gc.ca/census-recensement/2016/ dp-pd/prof/details/page.cfm?Lang=E\&Geo1=CD\&Code $1=3526 \& G e o 2=$ PR\&Code2 $=35 \&$ SearchText $=$ Niagara\&SearchType $=$ Begins \&SearchPR $=01$ $\& B 1=$ All\&GeoLevel=PR\&GeoCode $=3526 \& T A B I D=1 \&$ type $=0$. [cited 2020 Jun 16]

8. Michael Maclsaac. Ontario's emergency health services - sector overview. 2018. Available from: https://agendasonline.greatersudbury.ca/index. $\mathrm{cfm}$ ?pg=feed\&action=file\&agenda=report\&itemid $=4 \&$ id $=1278$

9. Channouf N, L'Ecuyer P, Ingolfsson A, Avramidis AN. The application of forecasting techniques to modeling emergency medical system calls in Calgary, Alberta. Health Care Manage Sci. 2007;10(1):25-45. https://doi.org/1 0.1007/s10729-006-9006-3.

10. Ambulance, Paramedics and Dispatch Operations - Niagara Region, Ontario. Available from: https://www.niagararegion.ca/living/health_wellness/ems/ operations.aspx. [cited 2020 Jun 9]

11. SAP BusinessObjects | Business Intelligence (BI) Platform \& Suite. SAP. Available from: https://www.sap.com/canada/products/bi-platform.html. [cited $2020 \mathrm{Jul} 7$ ]

12. Leeies M, Frrench C, Strome T, Weldon E, Bullard M, Grierson R. Prehospital application of the Canadian triage and acuity scale by emergency medical services. Can J Emerg Med. 2017;19(1):26-31. https://doi.org/10.1017/cem.2 016.345.

13. Niagara (Regional Municipality, Canada) - Population Statistics, Charts, Map and Location. Available from: https://www.citypopulation.de/en/canada/ ontario/admin/3526 niagara/. [cited 2020 Jun 16]

14. RStudio Team. R studio: integrated development for R. [internet]. Boston: RStudio, Inc:; 2019. Available from: http://www.rstudio.com/

15. Concerning 911 Trends for EMS Calls During COVID-19 Pandemic. JEMS. 2020. Available from: https://www.jems.com/2020/05/01/concerning-911trends-for-ems-calls-during-covid-19-pandemic/. [cited 2020 Jun 6]

16. WongLaura E, HawkinsJessica E, MurrellKaren L. Where are all the patients? Addressing Covid-19 fear to encourage sick patients to seek emergency care. NEJM Catalyst Innov Care Delivery. 2020.

17. Perlini S, Canevari F, Cortesi S, Sgromo V, Brancaglione A, Contri E, et al. Emergency department and out-of-hospital emergency system (112-AREU 118) integrated response to coronavirus disease 2019 in a northern Italy Centre. Intern Emerg Med. 2020;15(5):825-33. https://doi.org/10.1007/s1173 9-020-02390-4.

18. Lai PH, Lancet EA, Weiden MD, Webber MP, Zeig-Owens R, Hall CB, et al. Characteristics associated with out-of-hospital cardiac arrests and resuscitations during the novel coronavirus disease 2019 pandemic in New York City. JAMA Cardiol. 2020;5(10):1154-63. https://doi.org/10.1001/jamaca rdio.2020.2488. 
19. Onozuka D, Hagihara A. Extreme influenza epidemics and out-of-hospital cardiac arrest. Int J Cardiol. 2018;263:158-62. https://doi.org/10.1016/j.jjcard.2 018.02.028.

20. Shafi AMA, Shaikh SA, Shirke MM, Iddawela S, Harky A. Cardiac manifestations in COVID-19 patients-a systematic review. J Card Surg. 2020;35(8):1988-2008. https://doi.org/10.1111/jocs.14808.

21. Torales J, O'Higgins M, Castaldelli-Maia JM, Ventriglio A. The outbreak of COVID-19 coronavirus and its impact on global mental health. Int I Soc Psychiatry. 2020;66(4):317-20. https://doi.org/10.1177/0020764020915212.

22. Douglas M, Katikireddi SV, Taulbut M, McKee M, McCartney G. Mitigating the wider health effects of covid-19 pandemic response. BMJ 2020;369:m1557.

23. DeJong CAJ, DeJong Verhagen JG, Pols R, Verbrugge CAG, Baldacchino A. Psychological impact of the acute COVID-19 period on patients with substance use disorders: we are all in this together. Basic Clin Neurosci. 2020;11(2):207-16. https://doi.org/10.32598/bcn.11.covid19.2543.1.

24. City's busiest supervised injection site to reopen after month-long closure over COVID-19. thestar.com. 2020. Available from: https:/www.thestar.com/ news/city_hall/2020/04/16/citys-busiest-supervised-injection-site-to-reopen-a fter-month-long-closure-over-covid-19.html. [cited 2020 Jun 24]

25. Pabayo R, Alcantara C, Kawachi I, Wood E, Kerr T. The role of depression and social support in non-fatal drug overdose among a cohort of injection drug users in a Canadian setting. Drug Alcohol Depend. 2013;132(3):603-9. https://doi.org/10.1016/j.drugalcdep.2013.04.007.

26. Stoker S, McDaniel D, Crean T, Maddox J, Jawanda G, Krentz N, et al. Effect of shelter-in-place orders and the COVID-19 pandemic on orthopaedic trauma at a community level II trauma center. J Orthop Trauma. 2020;34(9): e336-e342.

27. Tourism. Niagara Economic Development. Available from: https://niagaraca nada.com/key-sectors/tourism/. [cited 2020 Jul 6]

\section{Publisher's Note}

Springer Nature remains neutral with regard to jurisdictional claims in published maps and institutional affiliations.

Ready to submit your research? Choose BMC and benefit from:

- fast, convenient online submission

- thorough peer review by experienced researchers in your field

- rapid publication on acceptance

- support for research data, including large and complex data types

- gold Open Access which fosters wider collaboration and increased citations

- maximum visibility for your research: over $100 \mathrm{M}$ website views per year

At $\mathrm{BMC}$, research is always in progress.

Learn more biomedcentral.com/submissions 